\title{
Ewing's Sarcoma of the Sacroiliac Joint Presenting as Tubercular Sacroiliitis: A Diagnostic Dilemma
}

\author{
Bhavuk Garg, Divesh Jalan, Prakash P Kotwal \\ Department of Orthopaedics, All India Institute of Medical Sciences, New Delhi, India
}

\begin{abstract}
We report a case of Ewing's sarcoma of the sacroiliac joint in a 21-year-old male mimicking tubercular sacroiliitis, a rare entity not reported in literature. He presented with pain in the lower back radiating to the right lower limb along with constitutional symptoms of 3 months duration. On examination, the right sacroiliac joint was tender. The laboratory investigations showed anaemia, leukocytosis and raised erythrocyte sedimentation rate. On X-ray, features of right sacroiliitis were seen. This was further investigated with magnetic resonance imaging (MRI), which showed features consistent with tubercular sacroiliitis. Patient was then started on antitubercular treatment, but the improvement was not consistent. So, a contrast MRI was done, which indicated features of primary sarcoma. It was then further confirmed by a computed tomography-guided biopsy, which showed features consistent with Ewing's sarcoma of the sacroiliac joint.
\end{abstract}

Keywords: Sacroiliac joint; Sacroiliitis; Ewing sarcoma; Magnetic resonance imaging

\section{Introduction}

Unilateral sacroiliitis is always seen as infective unless proved otherwise. Because of the widespread prevalence of tuberculosis in developing countries, this is usually considered as tubercular sacroiliitis. However, we encountered a diagnostic dilemma where unilateral sacroiliitis was managed on the lines of tubercular sacroiliitis after clinico-radiological assessment, which later proved to be Ewing's sarcoma.

Ewing's sarcoma may mimic osteomyelitis [1]. Ewing's sarcoma may present as mono-arthritis knee [2], spondylolisthesis [3] and postpartum back pain [4]. Moreover, Ewing's sarcoma of the ilium mimicking infectious [5] and inflammatory sacroiliitis $[6,7]$ are reported. However,
Ewing's sarcoma of sacroiliac joint mimicking tubercular sacroiliitis has not been reported in the literature to the best of our knowledge.

\section{Case Report}

A 21-year-old patient presented to us with low back pain of 3 months duration, which was moderate in intensity radiating down the right lower limb, aggravated by strenuous activity, prolonged standing and sitting and was relieved partially by lying down and analgesics. There was a history of low grade fever, malaise and loss of appetite. There was no history of trauma, known infection or contact with tuberculosis. There was no involvement of any other joints and rectal and urinary complaints.

Received Jul 9, 2012; Revised Oct 23, 2012; Accepted Oct 23, 2012

Corresponding author: Divesh Jalan

Department of Orthopaedics, All India Institute of Medical Sciences,

Ansari Nagar, New Delhi 110029, India

Tel: +91-99-99618797, Fax: +91-11-26588641, E-mail: Dvsh_Jalan@yahoo.com 
On examination, there was localized tenderness over the right sacroiliac joint. Sacroiliac provocation tests were positive. Lumbar spine and hip examination were normal. The left sacroiliac joint was also normal. The peripheral joints and neurological examination were normal as well. Investigations revealed an erythrocyte sedimentation rate of $45 \mathrm{~mm}$, haemoglobin of $10.8 \mathrm{~g} / \mathrm{dL}$ and total leucocyte count of 12,500 . The serum chemistry and urine analysis was normal. The chest radiograph was normal and tuberculin test was indeterminate ( $8 \mathrm{~mm}$ after 72 hours). Digital radiograph showed blurring, loss of cortical margins and juxta-articular sclerosis, suggestive of sacroilitis (Fig.1). Further investigation with magnetic resonance imaging (MRI) revealed abnormal marrow signal changes involving the right sacroiliac joint seen as T1 hypointense and STIR hyperintense marrow with intraosseous collection suggestive of infective sacroiliitis most likely tubercular in etiology (Fig. 2).

Based on the above findings, the diagnosis of tubercular sacroiliitis was made and the patient started on antitubercular therapy. After the 1-month follow-up, patient symptoms improved and objectively, erythrocyte sedimentation rate (ESR) came down to 9. The patient was continued on the same medication for the next 2 months. After 3 months, the patient complained of persistent pain with increase in intensity and the continuance of low grade fever off and on. At this time, a repeat MRI with contrast was done, which revealed a heterogeneous mass lesion involving the right iliacus, gluteal and paraspinal muscles with involvement of the right iliac wing, sacral ala and right sacroiliac joint (isointense on T1W and hyperintense on $\mathrm{T} 2 \mathrm{~W}$ with post contrast enhancement) (Fig. 3). Findings suggested a possibility of primary sarcoma or a lymphoproliferative disorder. At this time, a computed tomography (CT)-guided biopsy was conducted from the right sacroiliac joint, which showed a malignant small round cell tumor which was periodic acid-Schiff positive and immunopositive for CD99 (MIC 2) and focally for synaptophysin. Overall, the features were compatible with Ewing' sarcoma. Finally, the diagnosis of Ewing's sarcoma of the right sacroiliac joint was reached and the patient was started on multidrug chemotherapy. At the two-year follow-up, the patient's symptoms have significantly improved.

\section{Discussion}

Ewing's sarcoma accounts for approximately $10 \%$ of malignant bone tumors. The male: female ratio is 3:2. Approximately $90 \%$ of the affected patients are between 5 and 30 years [8]. Clinically, it presents as pain followed by swelling and a palpable mass [9]. Back pain and paraesthesias can be associated with paraspinal or pelvic tumors. Common nonspecific systemic manifestations include fever, fatigue, weight loss, and anemia mimicking acute or chronic infection, which needs to be considered in the differential diagnosis. Additional symptoms and signs depend on the specific location of the tumor. Ewing's sarcoma can affect all bones; however, it is slightly more common in tubular bones. The flat bones most often involved are the ilium, ischium, rib, and scapula. More than $50 \%$ of patients have a soft tissue component.

Despite such infections, particularly tuberculosis, being

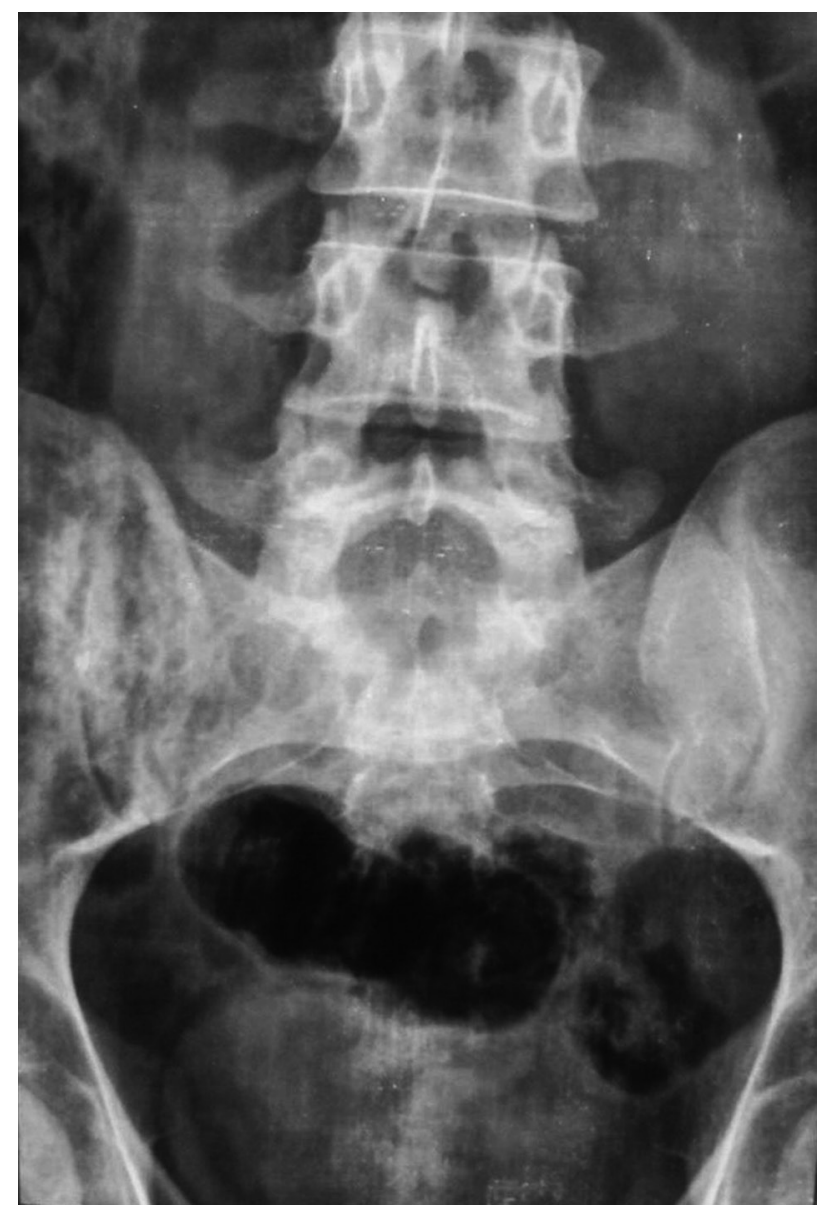

Fig. 1. Anteroposterior radiograph of the pelvis showing right sacroiliitis. The left sacroiliac joint is normal. 
very common in developing countries and the initial MRI also pointing to the infective pathology, an alternative diagnosis must always be thought in cases of unilateral sacroiliitis if the patient is not responding adequately to the treatment. The differential diagnosis of unilateral sacroiliac involvement should include infectious sacroiliitis, Reiter syndrome, psoriatic sacroiliitis and tumors, both primary and metastatic. The value of plain films of a sacroiliac region is compromised by the anatomy of the iliac bone and by the overlying structures. Therefore, MRI, computer tomography and bone scans are necessary [10]. $\mathrm{CT}$ is helpful in defining the tumor of Ewing's sarcoma by indicating the pattern of bone destruction and the associated soft-tissue mass [6]. MRI is more sensitive than $\mathrm{CT}$ in showing soft-tissue involvement and bone marrow metastases [5]. Definitive diagnosis is performed via open biopsy and histological examination combined with immunochemistry and cytogenetics.

Constitutional symptoms like fever, anemia and elevation of total leucocyte count, ESR and lactate dehydro-

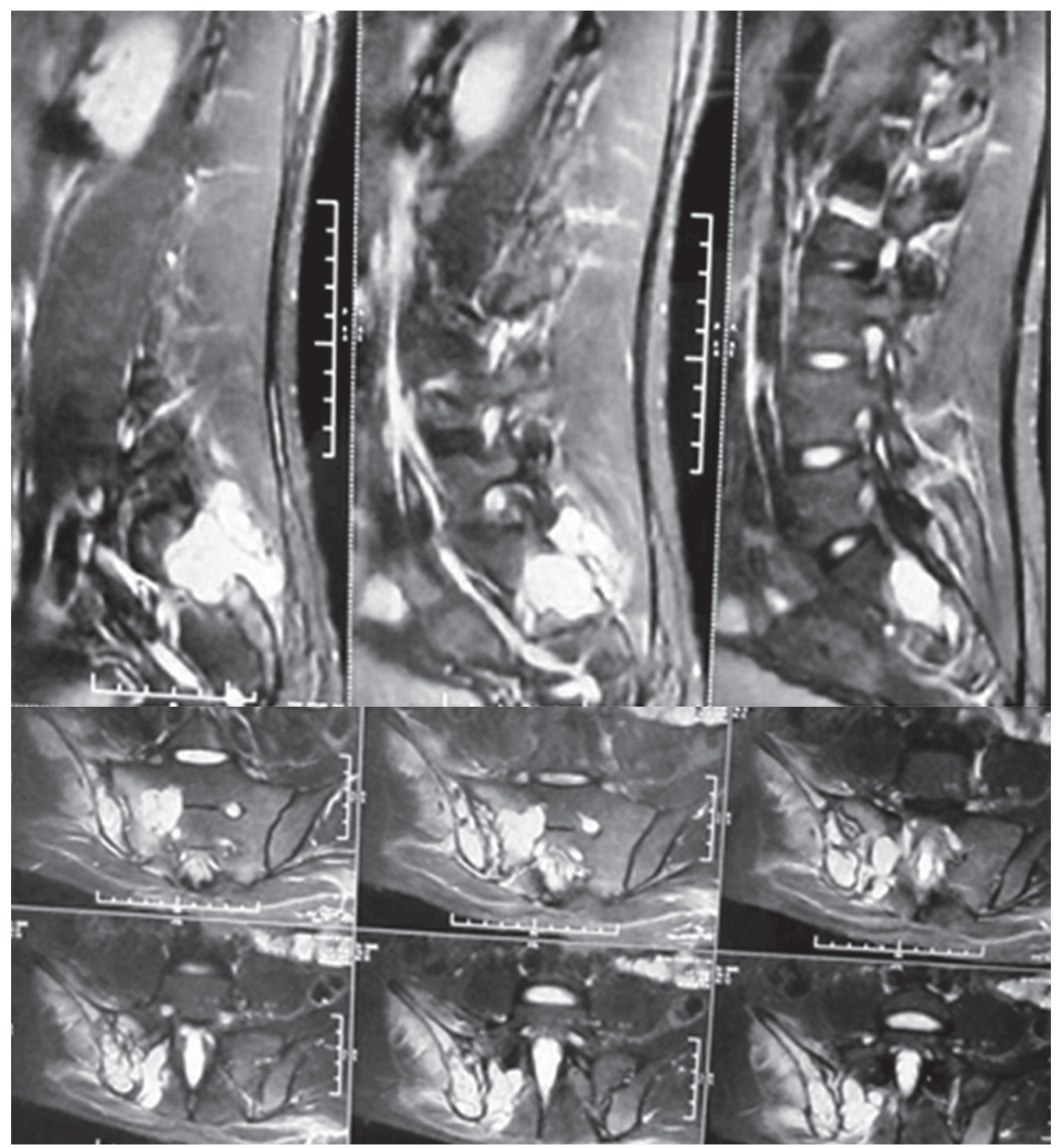

Fig. 2. Magnetic resonance imaging (sagittal and coronal sections) of the sacroiliac joint showing bright signal intensity around the right sacroiliac joint, suggestive of sacroiliitis. 


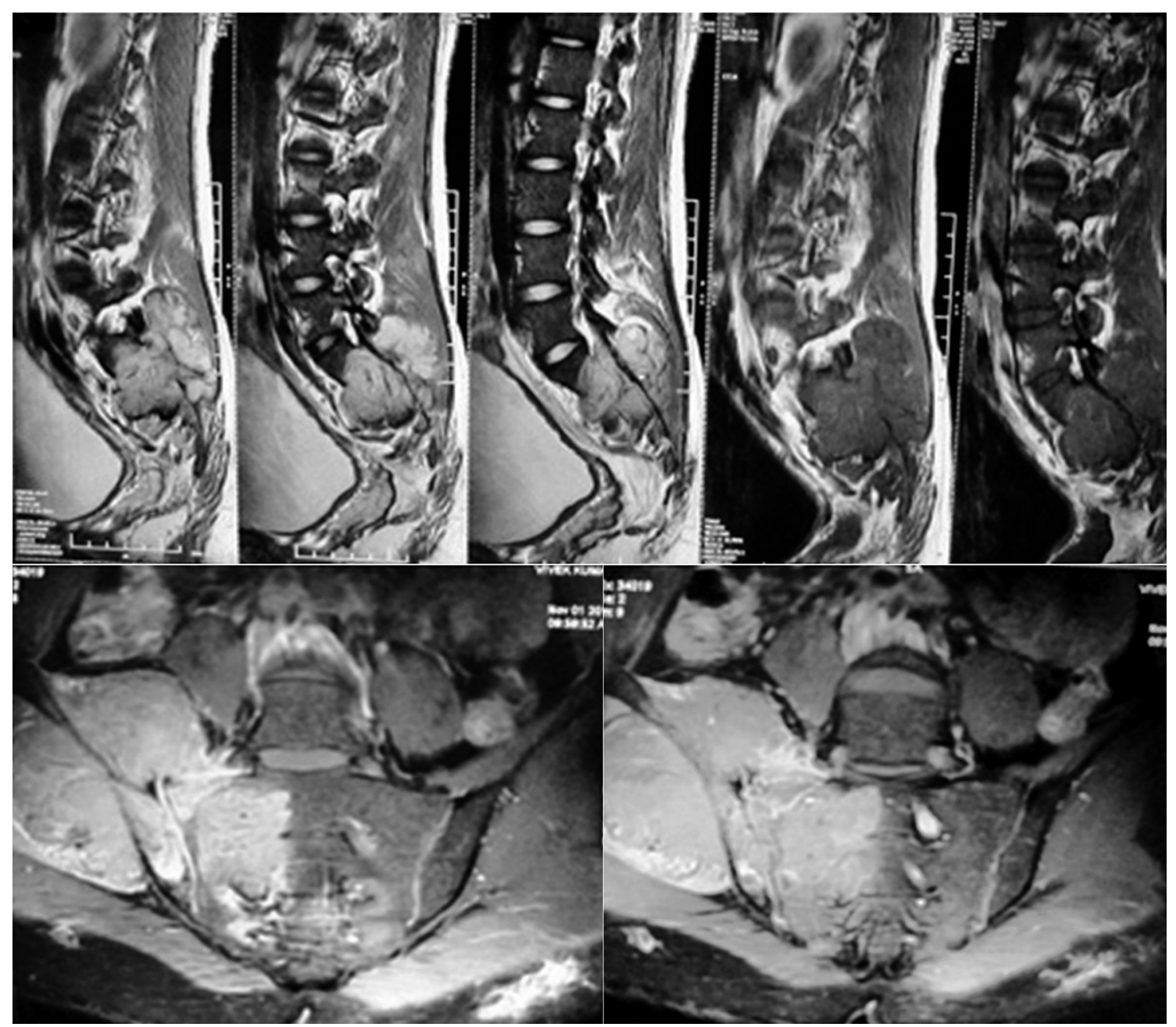

Fig. 3. Contrast enhanced magnetic resonance imaging (sagittal and coronal sections) obtained 3 months after starting antitubercular treatment showing a heterogeneous intensely enhancing mass involving the right iliac wing, sacrum, sacroiliac joint and the surrounding muscles suggestive of primary sarcoma.

genase can be seen in tuberculosis as well as in Ewing's sarcoma and should not be relied upon blindly. They have been reported to indicate more extensive disease and a worse prognosis.

The classical clinical and radiological presentation of Ewing's sarcoma of the iliac bone may not be the rule; one should be highly suspicious of the disease even if there is no direct pointer to the disease, as was encountered in our case. Biopsy and histopathological examination is often necessary when the clinical and radiological picture is ambiguous.

\section{Conflict of Interest}

No potential conflict of interest relevant to this article was reported.

\section{References}

1. Lyall HA, Constant CR, Wraight EP. Case report: Ewing's sarcoma in distal tibial metaphysis mimicking osteomyelitis. Clin Radiol 1993;48:140-2.

2. Halla JT, Hirsch V. Monoarthritis as the presenting manifestation of localized Ewing's sarcoma in an older patient. J Rheumatol 1987;14:628-31.

3. Klaassen MA, Hoffman G. Ewing sarcoma presenting as spondylolisthesis: report of a case. J Bone Joint Surg Am 1987;69:1089-92.

4. Mohl VK, Allerheiligen DA, Schoeber JK, Gee BD, Houston RE, Wildman K. Ewing's sarcoma presenting as postpartum back pain. Am Fam Physician 1996;54:878, 80, 82.

5. Pouchot J, Barge J, Marchand A, Carbonnier J, Vin- 
ceneux P. Ewing's sarcoma of the ilium mimicking an infectious sacroiliitis. J Rheumatol 1992;19:1318-20.

6. Adelman HM, Wallach PM, Flannery MT. Ewing's sarcoma of the ilium presenting as unilateral sacroiliitis. J Rheumatol 1991;18:1109-11.

7. Halwai MA, Mir BA, Wani MM, Bashir A, Hussain A. Ewing's sarcoma of the ilium mimicking inflammatory arthritis of the hip: a case report. Cases J 2009;2:6487.
8. Eggli KD, Quiogue T, Moser RP Jr. Ewing's sarcoma. Radiol Clin North Am 1993;31:325-37.

9. Vlasak R, Sim FH. Ewing's sarcoma. Orthop Clin North Am 1996;27:591-603.

10. Appell RG, Oppermann HC. Differential diagnosis of tumorous changes in the iliac bone region in children: case reports and review of the literature. Radiologe 1984;24:60-7. 